\title{
Novel beryllium-scintillator, neutron-fluence detector for magnetized liner inertial fusion experiments
}

\author{
C. L. Ruiz, ${ }^{1,}$ J. D. Styron, ${ }^{2}$ D. L. Fehl,${ }^{1, \dagger}$ K. D. Hahn, ${ }^{1}$ B. McWatters, ${ }^{1}$ M. A. Mangan, ${ }^{1}$ \\ G. W. Cooper, ${ }^{2}$ J. D. Vaughan, ${ }^{2}$ G. A. Chandler, ${ }^{1}$ B. M. Jones, ${ }^{1}$ J. A. Torres, ${ }^{1}$ S. A. Slutz, ${ }^{1}$ \\ D. J. Ampleford, ${ }^{1}$ M. R. Gomez, ${ }^{1}$ E. Harding, ${ }^{1}$ A. J. Harvey-Thompson, ${ }^{1}$ and P. F. Knapp ${ }^{1}$ \\ ${ }^{1}$ Sandia National Laboratories, Albuquerque, New Mexico 87131-0001, USA \\ ${ }^{2}$ University of New Mexico, Albuquerque, New Mexico 87185, USA
}

(Received 6 December 2018; published 22 April 2019)

\begin{abstract}
Progress in the development of a neutron detector employing the ${ }^{9} \mathrm{Be}(n, \alpha)^{6} \mathrm{He}\left(\beta^{-}, \tau_{1 / 2}=807 \mathrm{~ms}\right)$ reaction is reported. This detector's intended use is to estimate $2.45-\mathrm{MeV}$, deuterium-deuterium (DD) neutron yields from magnetized liner fusion (MagLIF) targets and proposed future gas-puff experiments at Sandia National Laboratory's $Z$ facility, Albuquerque, NM. This unique detector employs a simple stackedcomponent construction: 23 flat beryllium plates (99\% pure, $0.318 \mathrm{~cm}$ thick) sandwiched between 22 plastic scintillator sheets (BC-404, 1-mm-thick). Each Be plate and scintillator sheet is a planar square $(10 \mathrm{~cm}$ on a side) and the assembly is enclosed in a cubical, sealed, 1.5-mm-thick, Al-walled box. Scintillation light due to deposited energy from the ${ }^{6} \mathrm{He}$ betas is emitted and transmitted edgewise to ultraviolet-transmitting Lucite light guides optically coupled to two, independent, high-gain photomultiplier tubes. Reported here are details of the characterization of the neutron detection sensitivity (beta decay counts per incident neutron) of each photomultiplier tube side. These measurements were conducted at the Sandia National Laboratories ion beam facility, which provided beams of pulsed $175-\mathrm{keV}$ deuterons focused onto a 2.6-micron thick deuterated erbium target $\left(\operatorname{Er} D_{2}\right)$. Neutrons were produced via the $D(d, n)^{3} \mathrm{He}$ reaction. The neutron flux (incident neutrons $/ \mathrm{cm}^{2} / \mathrm{sec}$ ) on the Be detector was inferred by the measurement of the protons from the equally branched $D(d, p) T$ reaction. Steady-state neutron pulses incident on the Be detector induced the ${ }^{6} \mathrm{He}$ beta decay activity necessary to determine the detector's neutron sensitivity referred to as the $F$-factor. The rational, methodology, and experimental details behind this approach and the results with uncertainties of the subsequently determined $F$-factor of the Be detector are detailed. Additional comments regarding improvements to the calibration methodology plus future studies and the use of this detector in $Z$ experiments will be discussed briefly.
\end{abstract}

DOI: 10.1103/PhysRevAccelBeams.22.042901

\section{INTRODUCTION}

Ongoing physics experiments at the $Z$ facility (Sandia National Laboratory, New Mexico) are presently evaluating the magnetized liner inertial fusion (MagLIF) concept for inertial confinement-fusion studies with $D_{2}$-filled targets [1]. Essential to making this assessment is a reliable and precise measurement of the, deuterium-deuterium (DD) neutron yield $Y_{\mathrm{DD}}$ which is a quantity that can be compared

\footnotetext{
*lruiz@Sandia.gov

Retired Sandia National Laboratories, Albuquerque, New Mexico, USA.

Published by the American Physical Society under the terms of the Creative Commons Attribution 4.0 International license. Further distribution of this work must maintain attribution to the author(s) and the published article's title, journal citation, and DOI.
}

with model predictions and serve as a monitor of shot-toshot fusion yield variations.

In the past, nuclear activation has been a simple, passive technique to diagnose neutron yield in such experiments. Presently at $Z$, the adopted, standard diagnostic for measuring DD neutron yields is the activation of indium $\left({ }^{115} \mathrm{In}\right)$ : specifically, the ${ }^{115} \operatorname{In}\left(n, n^{\prime}\right)^{115 m} \operatorname{In}$ reaction with a $0.38 \mathrm{MeV}$ threshold and an isomeric daughter nucleus $\left({ }^{115 m} \mathrm{In}\right)$ that emits a $336.23 \mathrm{keV}$ gamma ray with a $4.49 \mathrm{hr}$ half-life. The initial activity after a $Z$ shot is a measure of $Y_{\mathrm{DD}}$. Details for this diagnostic, its characterization using a similar methodology, and application to $Z$ have been published elsewhere $[2,3]$.

We have been challenged to find an independent and complementary approach to estimating neutron yields at $Z$. The goals were to diminish activation from scattered neutrons by moving away from the source into the far radiation field and to provide an in situ means of detecting and counting activated nuclear decays in a much shorter 
and immediate timescale (minutes after the experiment) to report DD yields.

We report here the construction, characterization, and sensitivity of a novel neutron-yield detector compatible with the $Z$ environment and, specifically, to MagLIF, DD pulsed neutron experiments. Guided by previously published designs [4-6], the new diagnostic is based on the ${ }^{9} \mathrm{Be}(n, \alpha){ }^{6} \mathrm{He}$ reaction which has a neutron energy threshold of $0.67 \mathrm{MeV}$ and a total reaction cross section of $82.5 \mathrm{mb}$ at $2.45 \mathrm{MeV}$ [7]. Principally, beta electrons (3.5-MeV-end point) from decaying ${ }^{6} \mathrm{He}\left(\tau_{1 / 2}=0.807 \mathrm{~s}\right.$ half-life, branching ratio 0.9999) [8] from activated Be are absorbed in an adjacent plastic scintillator with subsequent visible light pulses produced and viewed by a photomultiplier tube (PMT) and counted.

The organization of this article is as follows. Section II describes the operating principles of the detector and principal design studies of optimum neutron sensitivities from Monte-Carlo simulations. Section III details the configuration of the presently characterized detector derived from these studies. Section IV summarizes how the neutron sensitivity, or the $F$-factor (counts/per incident neutron/ $\mathrm{cm}^{2}-\mathrm{sec}$ ), of the Be detector was measured from a known, pulsed, DD neutron source. Included in this section are the experimental setup, data collection, and the activation decay analysis as a function of PMT bias and counting electronics parameters. The overall results of these characterizations (with uncertainties) are given in Sec. V. Section VI briefly describes the application of this detector to MagLIF DD experiments, improvements to the associated-particle techniques (APT), future DT neutron sensitivity studies, precalibration checks, and concluding remarks. To emphasize the technique and formulation there is an attached Appendix with mathematical and physical descriptions of the $F$-factor determination and the associated particle technique used to infer neutron fluence production.

\section{BASIC OPERATING PRINCIPLES OF THE BE-SCINTILLATOR DETECTOR FOR DD NEUTRONS AT $Z$}

Figure 1 sketches a simple detector concept in one dimension for a single pair of Be scintillator layers, shown edgewise with normal vectors pointing vertically away from the neutron source. In this illustration, the upper layer is beryllium with a thickness $\Delta Z_{\mathrm{Be}}$, and the bottom layer is a plastic scintillator, thickness $\Delta Z_{\mathrm{SC}}$. A complete neutron detector consists of $N$ such layered pairs with an overall length of $Z_{\mathrm{TOT}}=N Z_{1}$. It is assumed that the top face of the Be-scintillator stack is some distance from the neutron source.

Figure 1 also indicates various particle interactions, due to a mixed-field radiation source (arrows A-E) produced by MagLIF experiments, which occur in a Be-scintillator pair.
For example, prompt, hard, gamma rays $\mathrm{A}, \mathrm{B}(h \nu \gtrsim$ $0.5 \mathrm{MeV}$ ) interact via Compton and pair-production processes in both detector layers depositing energy in the scintillator and producing prompt $(\sim \mathrm{ns})$ light output signals detected by the PMT. Similarly, DD neutrons (Fig. 1, arrow C) from the source arrive at the detector simultaneously and directly interact with the scintillator by elastic $\mathrm{n}$ - $\mathrm{p}$ processes, leading again to prompt, proton-induced light output signals.

But more to the point, Fig. 1 also shows the neutron interaction of interest (Fig. 1, arrow D), ${ }^{9} \mathrm{Be}(n, \alpha)^{6} \mathrm{He}$, and the subsequent route by which the activation information moves toward detection: (a) a fraction of the emitted betadecay electrons from ${ }^{6} \mathrm{He}$ escape the Be layer, pass the Be-scintillator boundary, and individually deposit an energy dose in the scintillator; and (b) a fraction of each dose is converted in the scintillator to a visible light pulse, converted by the PMT to a 20 ns wide signal and singleevent counted long after (millisecond timescales) the prompt radiation has passed.

Optimizing the efficiency of the detector is an exercise in optimizing the geometrical parameters for this geometry [9]. The expected MFP (mean-free path) for DD neutrons is 3.4 and $4.7 \mathrm{~cm}$ in beryllium and scintillator respectively. Thus, from a simple, exponential, uncollided flux point of view [10-14] one may expect the Be-scintillator detector to have an overall linear dimension $\sim 10 \mathrm{~cm}(\sim 3 \mathrm{MFP})$ with

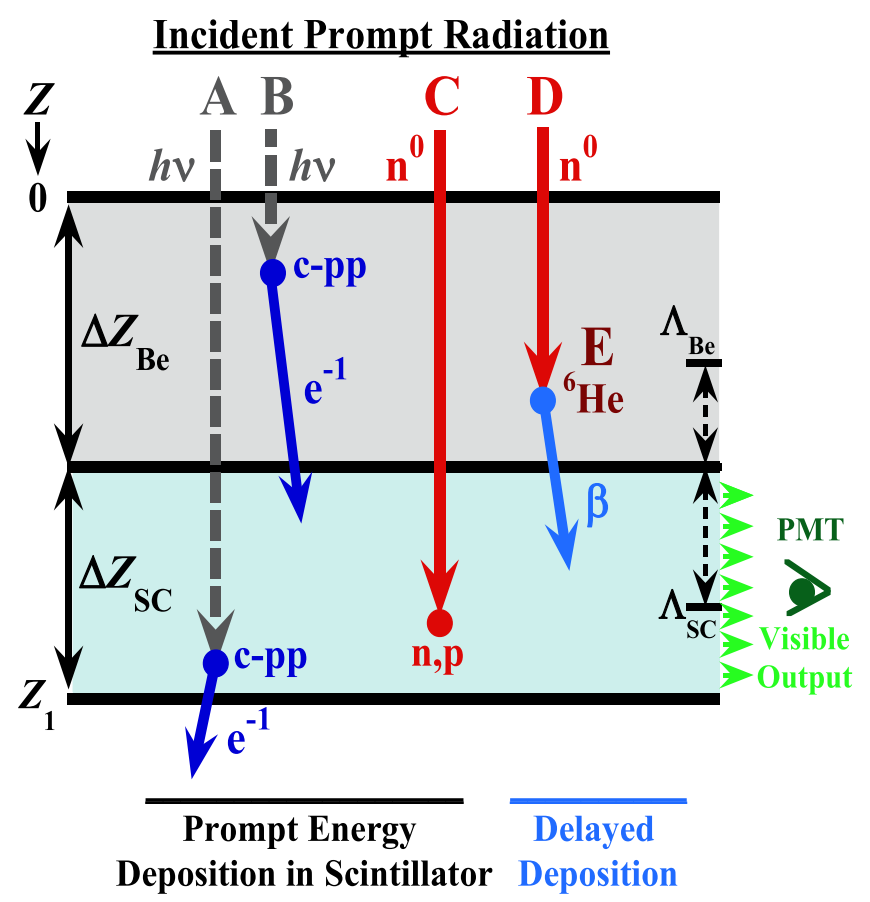

FIG. 1. Individual $n^{0}, \gamma, \beta$-interactions in a Be-BC404 (SC) detector pair. Prompt and delayed energy processes are indicated in the scintillator (although backscattered results are not shown). Thickness parameters, $\Delta Z_{\mathrm{Be}}$ and $\Delta Z_{\mathrm{SC}}$, together with their corresponding $\beta$-ranges $(\Lambda)$ are sketched (but not to scale). 
some tens of paired Be and scintillator layers, each a few $\mathrm{mm}$ thick since the average range for a nominal ${ }^{6} \mathrm{He}$ beta decay electron $(1.2 \mathrm{MeV})$ is 0.28 and $0.49 \mathrm{~cm}$ for beryllium and scintillator respectively.

Realistic estimates of the detector response for several geometric permutations were investigated using the Monte Carlo code MCNP [15]. A fixed detector volume was chosen $\left(10 \mathrm{~cm} \times 10 \mathrm{~cm}\right.$ surface, $Z_{\mathrm{TOT}}=10.4 \mathrm{~cm}$ height) such that $Z_{\text {TOT }}$ was held fixed at $10.4 \mathrm{~cm}$ as $\Delta Z_{\mathrm{Be}}$ and $\Delta Z_{\mathrm{SC}}$ were varied in thickness $-0.25,0.50,0.75$, $1.00,2.00$, and $3.00 \mathrm{~mm}$ (a total of 36 possible geometric permutations). In each case, there was an extra scintillator layer on top to pick up backward emitted beta electrons from the Be layer of the first layered pair. A lumped figure of merit, or detector efficiency, $\eta\left(\Delta Z_{\mathrm{Be}}, \Delta Z_{\mathrm{Be}}\right)$ was calculated using the product of MCNP FM4 and F8 tallies as shown in Fig. 2. The product defines the fraction of incident neutrons that ultimately produce beta energy losses in the scintillator greater than $10 \mathrm{keV}$ (expected minimum where signals may be measurable).

The FM4 tally, which is a measure of the ${ }^{6} \mathrm{He}$ nuclei produced in each Be layer, incorporates the energy dependent neutron flux, the energy dependent ${ }^{9} \mathrm{Be}(n, \alpha)^{6} \mathrm{He}$ cross section, the Be atom density, and the volume of each layer. This was calculated by propagating neutrons through the detector geometry using an isotropic, point source of 2.45 MeV DD neutrons located $50 \mathrm{~cm}$ directly above the detector stack. The F8 tally is a measure of the beta decay pulse-height energy spectrum and is found by

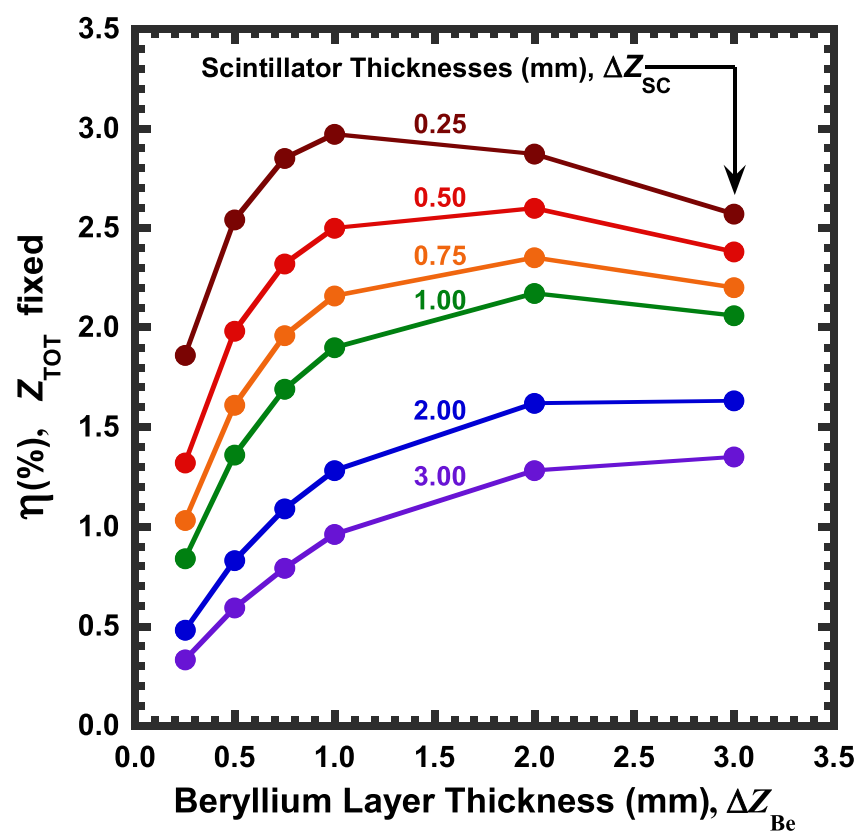

FIG. 2. Estimated counting efficiency for a fixed dimension beryllium/scintillator layer detector as a function of beryllium and scintillator thicknesses from coupled neutron/beta transport calculations in MCNP6. summing the response of each scintillator region. This was calculated using an isotropic beta electron source term with a Fermi energy spectrum of end point $3.5 \mathrm{MeV}$ [16] that was randomly and uniformly distributed within each Be layer (proportional to the number of ${ }^{6} \mathrm{He}$ nuclei produced in each Be layer from the first calculation). The default electron cutoff energy of $1 \mathrm{keV}$ was implemented. Enough sample MCNP trajectories were taken for each parameter combination to estimate $\eta$ with an uncertainty $\sim 1 \%$ (at $95 \%$ confidence). Figure 2 shows $\eta$ estimated by MCNP for a stacked Be detector of $N$ Be-scintillator pairs. One sees a family of curves that rise from low values to a broad peak, and then a decrease. The general shape of these curves is based on the trade-off between increasing the activation (left of the peak) and increased electron stopping (right of the peak).

While the results shown in Fig. 2 provided insight into how the efficiency would change with geometric design, the design of the Be-scintillator stack used for $Z$ experiments was determined experimentally. A prototype was built with 2-mm thick scintillator sheets and tested on several MagLIF experiments. So much light output from captured prompt radiation was recorded such that the decay counts were unusable for several 100's of milliseconds, a time span which overlapped the desired early-time counts due to ${ }^{6} \mathrm{He}$ decay. The scintillator thickness was then reduced to $1 \mathrm{~mm}$, and this problem no longer obtruded. The overall result of these studies was that (a) the detector and counting equipment survived; and (b) after prompt radiation had passed the detector, the output counting signal of the Be-scintillator was directly proportional to the remaining activity (counts/unit time) of the ${ }^{6} \mathrm{He}$ as a function of time but with a steady background count rate.

A result from a separate study [9], using the scintillator thicknesses modeled, showed that scintillators $<1 \mathrm{~mm}$ have a high counting efficiency at low discriminator levels $(<20 \mathrm{mV})$, however, the thin scintillator limits the amount of energy that the beta particles can deposit; limiting the signal amplitude of the measured signal and the desired dynamic range. Therefore, a 1-mm thick scintillator was deemed the most feasible choice for a Be detector design for use at the $Z$ facility.

\section{CONFIGURATION OF THE BE DETECTOR}

Guided by MCNP modeling and preliminary testing on $Z$, the final design for the Be-scintillator neutron detector chosen for characterization is shown in Fig. 3. Shown are the radiation sensitive "active area" and ancillary, visiblelight collecting components.

The active region is a cube (10 $\mathrm{cm}$ on a side) consisting of vertically stacked, alternating layers of $3.18 \mathrm{~mm}$, $99.9 \%$ atomic purity $\mathrm{Be}$ (22 total) and $1 \mathrm{~mm}$ thick, BC-404 plastic scintillator [17] layers (23 total) normal to a neutron source. Each scintillator layer is faced with $100-\mu \mathrm{m}$-thick, aluminized Mylar films - the aluminum side 


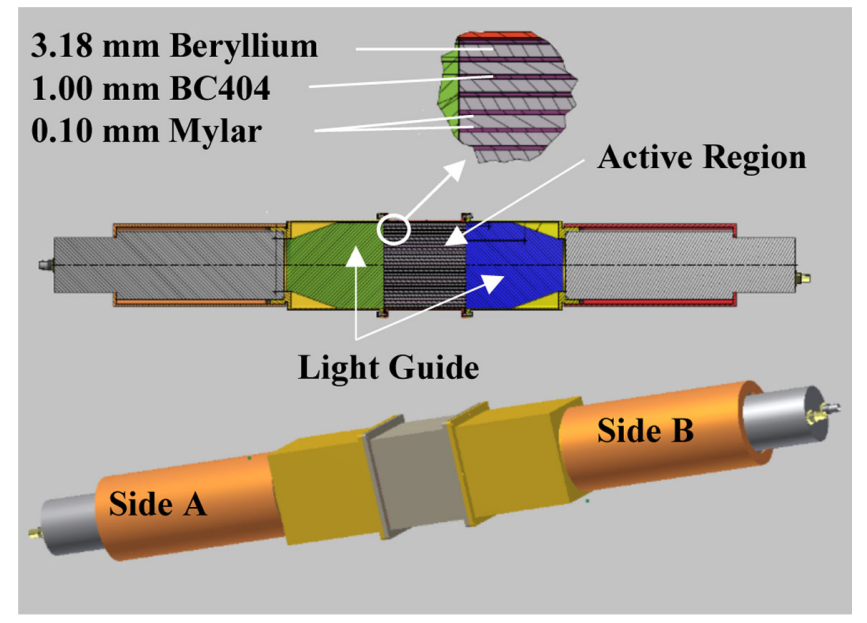

FIG. 3. Be detector configuration with outputs A and B. Optical pulses from the Be-BC404 stack are viewed by two independent PMTs at side A and B (shown in gray).

facing the scintillator is to reflect scintillator light that would otherwise be lost due to internal absorption at the interface. The active region is encased within a cubical, aluminum box (1.5 mm-thick walls) with two opposite ends open so that the scintillator emissions over the entire 10 by $10 \mathrm{~cm}$ area can be viewed by contact-coupled, ultraviolet-transmitting (UVT) Lucite light guides. These light guides are tapered to mate with the $7.62-\mathrm{cm}$ diameter photocathodes of two, separate, inline Hamamatsu model R6091 PMTs (denoted sides A and B, respectively) [18]. In this arrangement, one obtains two independent ${ }^{6} \mathrm{He}$-decay signals for comparison and backup should either channel A or B be lost.

\section{EXPERIMENTAL CHARACTERIZATION OF THE SNL BE DETECTOR}

\section{A. Qualifying a continuous DD-neutron source with the associate-particle technique (APT)}

We have used a particle accelerator to create a DD neutron source of sufficient fluence to allow measurements of the Be detector neutron sensitivity. The neutron source fluence determination is detailed in the Appendix. In the present application, energetic deuterium ions are accelerated onto a deuterium-loaded target to produce the DD reactions with nominal $2450 \mathrm{keV}$ neutrons. The $D(d, n)^{3} \mathrm{He}$ and $D(d, p) T$ reactions have a branching ratio of nearly $1: 1$ [19]. A measurement of either ${ }^{3} \mathrm{He}, p, T$ or all is sufficient to infer neutron production. This method is called the associated-particle technique (APT) and is described in detail in the Appendix.

The DD reactions were produced at Sandia's Ion Beam Laboratory (IBL). A $300 \mathrm{kV}$ Cockroft-Walton accelerator provided a stable, $d^{+}$deuteron beam with a kinetic energy of $175 \mathrm{keV}$ ( $\pm 1 \mathrm{keV}$ energy resolution). The ion beam was magnetically analyzed to remove any molecular deuterium $\left(d_{2}^{+}\right)$in the beam. There was also an electrostatic sweep circuit upstream capable of moving the beam onto and off target with a timing uncertainty of $0.1 \mathrm{~ms}$ to allow for a well-timed pulsed neutron source. An electrostatic quadrupole lens then focused the $d^{+}$beam onto a deuteriumloaded target. The deuteron beam current incident on a target was adjustable between 0.5 and $6.0 \mu \mathrm{A}$.

The target for the $d^{+}$beam was located at the center of a $15.24 \mathrm{~cm}$ diameter, $0.318 \mathrm{~cm}$ thick vacuum chamber (a cutaway drawing is shown in Fig. 4) and consisted of a 2.6- $\mu \mathrm{m}$ thick, $1-\mathrm{cm}$ diameter film of erbium coated onto a $625-\mu \mathrm{m}$-thick, 1.9-cm-diameter, oxygen-free copper substrate and loaded with deuterium at an atomic ratio of 1.98 (i.e., $\sim 2$ deuterium atoms to each erbium atom). This $\operatorname{Er} D_{2} /$ copper target was mounted onto an electrically isolated, copper, cold finger (either air or water cooled), a precaution to prevent overheating of the target and the subsequent outgassing of the $D_{2}$ from the substrate-a process that both degrades neutron production and yields a time-dependent output. In addition, a cylindrical, $0.25-\mathrm{mm}-$ thick tantalum cover, biased at $-80 \mathrm{~V}$ relative to the target, surrounded the $\operatorname{Er} D_{2}$ target to suppress secondary electrons produced by the impinging $d^{+}$beam from leaving the target surface (cf. Fig. 4, and below). Doing so allowed for a reliable current measurement of beam on target with an ORTEC 556 current integrator.

Two, 200- $\mu \mathrm{m}$ thick, $25 \mathrm{~mm}^{2}$ active area, silicon surfacebarrier ORTEC detectors (SBD) were chosen to measure the associated charged particles [20]. As shown in the bottom panel of Fig. 4, the SBDs were located $29.2 \mathrm{~cm}$ from $\operatorname{Er} D_{2}$ target center at $110.0^{\circ}$ and $165.0^{\circ}$ relative to the incoming $d^{+}$beam direction at $0^{\circ}$. The normal of the target was adjusted to a midangle of $137.5^{\circ}$ such that each SBD detected the emitted associated charged particles with nominally the same range in the $\operatorname{Er} D_{2}$. Each SBD had in front a 5-mm diameter, 10 mill thick Ta collimator to define the solid angle subtended relative to the source. The collimator openings were also covered with $2.7 \mu \mathrm{m}$-thick, pinhole-free, aluminum foils to range out low-energy Rutherford scattered $d^{+}$nuclei from the target surface.

Considerable care was taken to align the SBDs with the target and to define the solid angle between the source and the SBDs. Several techniques were used to check this. First, the collimators and alignment parts were manufactured with tight tolerances. Second, the $d^{+}$-beam profile on target was determined by substituting a similarly positioned quartz target, which would fluoresce allowing the beam profile $(\sim 0.25-\mathrm{cm}$ diameter) to be ascertained with a camera. Third, besides metrological measurements which gave $(2.177 \pm 0.022) \times 10^{-4}$ sr for each $\mathrm{SBD}$, the solid angles were checked experimentally. A $1-\mu \mathrm{Ci}{ }^{241} \mathrm{Am}$ $\alpha$-emitting source [21] (total activity calibrated within $\pm 3 \%$ 1-sigma uncertainty) was placed at the same surface location as the $\operatorname{Er} D_{2}$ target. Its effective active diameter was $0.25 \mathrm{~cm}$ for $\alpha$ emission (corresponding to the beam 


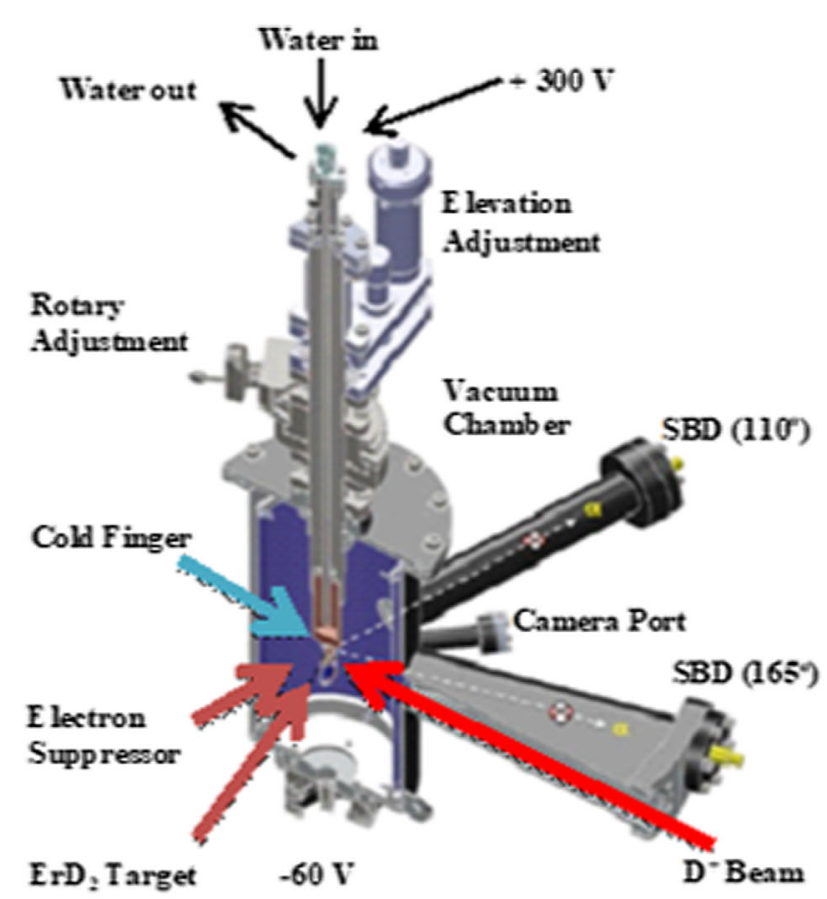

(a)

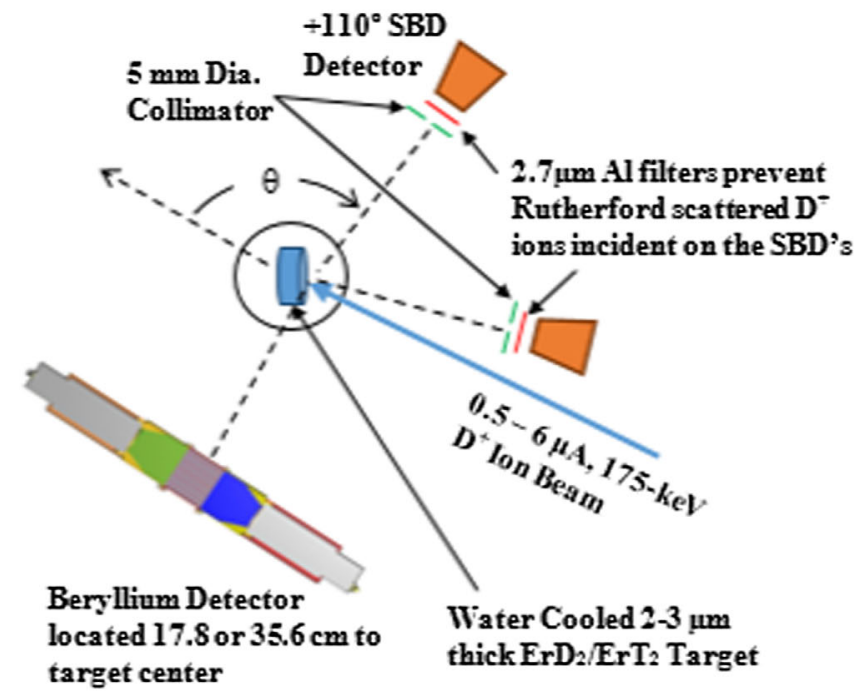

(b)

FIG. 4. (a) Experimental APT vacuum chamber. (b) Associatedparticle plus Be detector experimental layout. The angle $\theta$ is either 110 or 165 degrees. The 5 -mm diameter, Ta collimators define the SBDs subtending solid angles.

diameter), which was detected by the SBDs and used to determine each solid angle. The result was $(2.258 \pm$ $0.068) \times 10^{-4} \mathrm{sr}$, which compares favorably with the metrological measurements. The $\alpha$-source-determined solid angles were used in all subsequent data analysis here, with an estimated 1-sigma uncertainty of $\sim \pm 3 \%$.

The associated-particle signals from the SBDs were preamplified by ORTEC 142A modules, then amplified by ORTEC 672 amplifiers whose unipolar outputs were

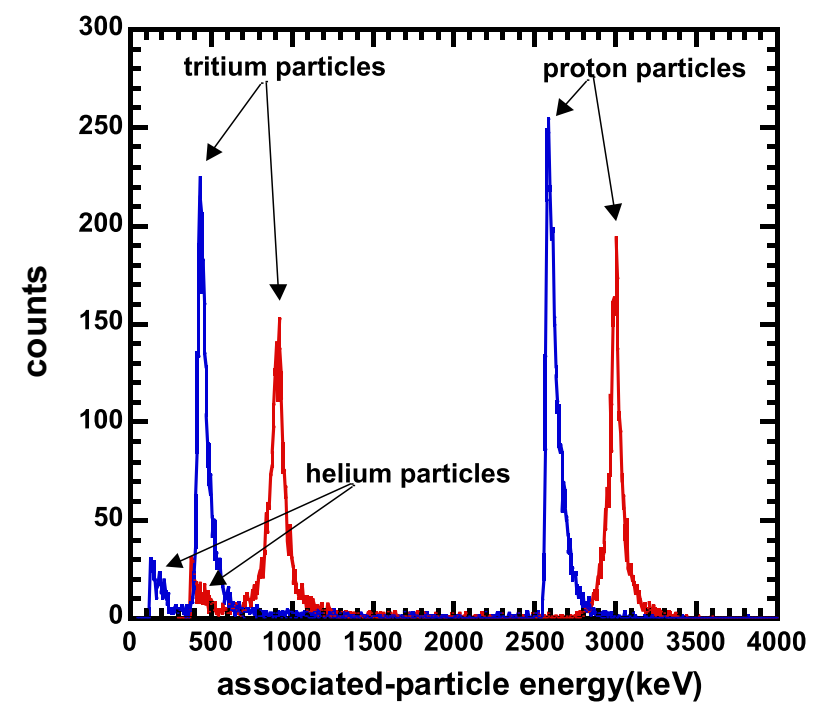

FIG. 5. Associated-particle energy spectra from the 165 and 110 degrees SBDs for the equally branched DD reactions: $D(d, n)^{3} \mathrm{He}$ and $D(d, p) T$. Blue spectra pertain to the 165 -deg detector. Red spectra pertain to the 110-deg detector.

routed to separate ORTEC multichannel analyzers. This nucleonic scheme allowed monitoring of the entire tritium and proton charged-particle energy spectra, and the highest-energy portion of the lower energetic ${ }^{3} \mathrm{He}$ ions. For example, Fig. 5 displays a particle energy spectrum measured at SBD locations $110^{\circ}$ and $165^{\circ}$, respectively. Having two SBDs at the $110^{\circ}$ and $165^{\circ}$ positions (see the Appendix) provided two measurements to infer neutron fluence. In each plot, the highest energy peak (in channel numbers) corresponds to protons (2600-3000 channels), the middle peak by tritons (500-900 channels), and the lowest partial peak to ${ }^{3} \mathrm{He}$ nuclei $(<500$ channels). Protons were ultimately chosen as the associated particle to determine the inferred neutron production. For redundant particle monitoring, the bipolar outputs from the amplifiers were routed to single-channel analyzers (SCAs) for setting upper and lower level discrimination of only the proton peak. The logic TTL outputs from these SCAs were routed to multichannel scalars (ORTEC Easy-MCS).

Besides using two SBDs to monitor proton counts, a third measurement monitored the current stability and constancy of the incoming $d^{+}$beam. It was important to keep the $d^{+}$beam on target and close to a steady-state current to avoid time-dependent neutron-activation-rate corrections (cf. the Appendix).

Lastly, in Fig. 6 we show a correlation plot of the neutron flux inferred by associated-particle proton measurements at 165 and 110 degrees. The data indicate consistently the agreement of inferred yield from the 165- and 110-degree charge-particle detectors. Taking all these measurements together, one argues that the target-assembled IBL accelerator experiments provide an appropriate and stable source of DD neutrons for characterizing the Be-based scintillator neutron detector. 


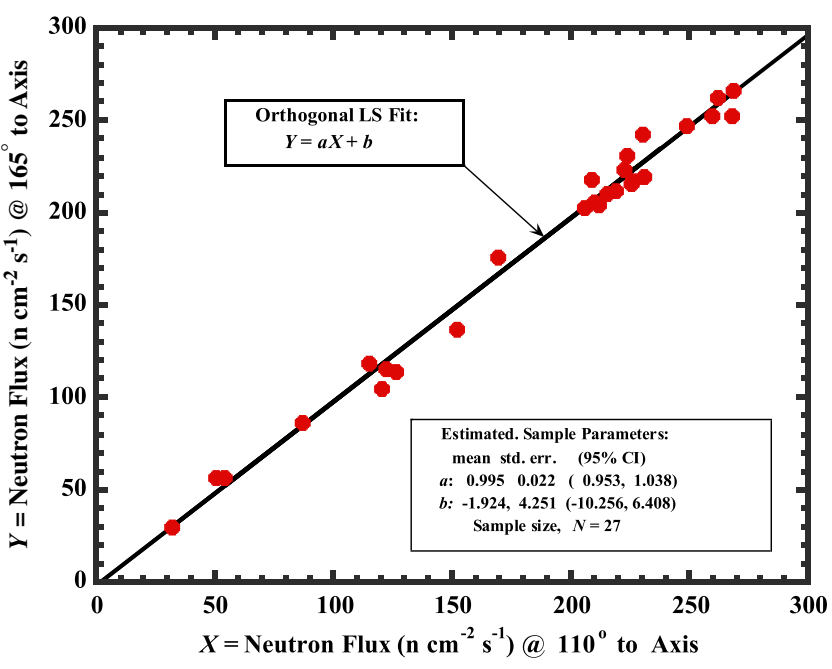

FIG. 6. Correlation plot between neutrons inferred between the AP measurements at 110 and 165 degrees for 24 experimental DD runs.

\section{B. Be detector measurement procedures and experimental setup}

At $175 \mathrm{keV}$ incident-energy, kinematic calculations suggest that the Be detector's center-normal $(10-\mathrm{cm}$ by $10-\mathrm{cm})$ face be placed at 95.0 degrees relative to the beam direction to subtend $2450 \mathrm{keV}$ DD neutrons. The PMTs, chosen for their high gain $\left(5 \times 10^{6}\right)$, linearity, and low dark-noise characteristics were placed on opposite sides of the Be detector component to detect scintillator signals. These tubes have 7.6-cm diameter photocathodes and linear-focusing dynode grids; they were connected to appropriate Hamamatsu resistive divider-chain bases. The high-voltage bias for each PMT was supplied by Stanford 336's power supplies [22]. Signals from each PMT were fed directly through $50-\Omega$, RG-223 Co-Ax cable into output-signal-adjustable ORTEC 584 constant-fraction discriminators (CFDs). The negative outputs of the discriminators were then directed to ORTEC EasyMCS modules for single-event-rate counting.

Neutron production from the target was monitored by gating a four-second deuteron beam-on-target pulse with $\leq 0.1$-ms gating uncertainty. This uncertainty was due to the delay of switching on the current from a power supply connected to electrostatic deflection plates steering the beam onto the downstream $\operatorname{Er} D_{2}$ target. Furthermore, during beam-on-target time, the variation in beam current was less than $0.5 \%$, and, thus, time-dependent, activation corrections were unnecessary. During the irradiation time, $t_{0}$, both buildup and decay of ${ }^{6} \mathrm{He}$ is taking place and the activity is not counted. In addition, if copious counts due to neutrons and prompt-gamma rays interacting in the scintillator are observed during $t_{0}$ would invalidate any measurements during this time interval. A similar result could also occur due to the 0.1 -ms beam-off-target uncertainty overlapping the beginning of the ${ }^{6} \mathrm{He}$ decay activity. This

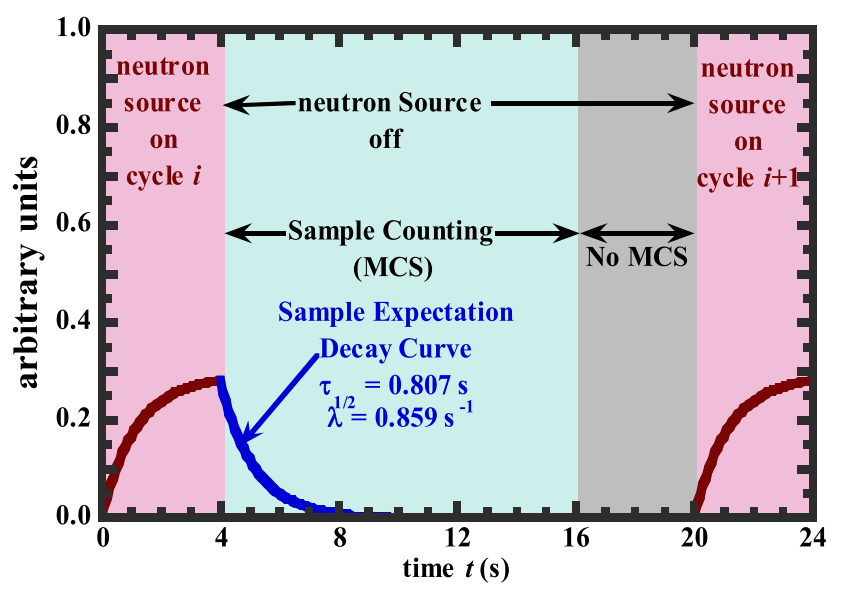

FIG. 7. Irradiating counting and gating scheme in one $i$ th cycle. The neutron irradiation time period of $4 \mathrm{~s}$ is in the pink region. The counting of Be decay is for $12 \mathrm{~s}$ and in green. The gray area indicates no-counting for $4 \mathrm{~s}$. A complete $i$ th cycle repeated every 20 seconds for a minimum of 120 cycles.

effect was not observed here. Therefore, only the decay of the ${ }^{6} \mathrm{He}$ induced activity after $t_{0}$ was absolutely counted for a fixed time counting interval between times $t_{1}$ and $t_{2}$. Because of the $0.807 \mathrm{~s}$ half-life decay of ${ }^{6} \mathrm{He}$ and potential equilibrium (activation buildup and decay activity become equal), it was not advantageous to induce activation for too many half-lives by increasing the $t_{0}$ neutron irradiation. Therefore, a practical four-second or less irradiation time $\left(t_{0}=5\right.$ half-life) was chosen. As mentioned, particle spectra were collected only during beam-on time. At the end of four seconds the beam-on gate was turned off to stop the beam impinging on the target and a separate gate turned on (within 0.1-ms) to trigger the Easy-MCS's for a 12 -second ${ }^{6} \mathrm{He}$ decay counting interval. The Easy-MCS's were set to a 0.1-second dwell time (time bin). The counting was stopped by a gate at the end of 12 seconds allowing for a four-second period where no counts were recorded, although this was arbitrarily decided.

The entire time gating scheme, as depicted in Fig. 7, for each four-second beam-on interval was repeated every 20 seconds for a preselected number of beam-on-plusdecay counting periods. This gating scheme was controlled to submillisecond timing by a programmable Stanford 648 delay-gate generator. For the four-second interval that the current was on target the neutron production was minimal and resulted in low count rates during the 12-second counting interval. It was found that with a $2-\mu \mathrm{A}$, beam current, 120 beam pulses were necessary to obtain statistically significant decay data, which required 40 minutes of operating time. Several longer distances between the detector and the neutron source were also studied. These longer distances required 360 or more pulses to obtain good statistics. Data from the Be detector labeled No. 2 was collected with the following geometric parameters: the front center face of the detector was located either 178 or 


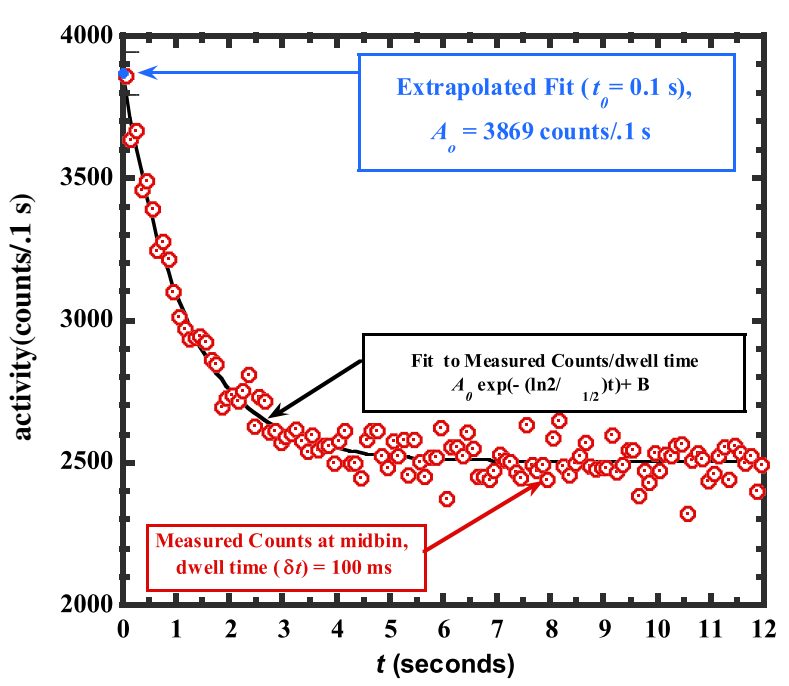

FIG. 8. The decay curve for the Be neutron detector in run 64 (side A), with a three-parameter, nonlinear, weighted leastsquares fit. The extrapolated initial point $A_{0}+B$ at $t_{0}$ is estimated here to be 3869 counts in $100 \mathrm{~ms}$.

$356 \mathrm{~mm}( \pm 2 \mathrm{~mm})$ from the $\operatorname{Er} D_{2}$ target at $95^{\circ}$ relative to the $0^{\circ} d^{+}$beam.

Multichannel scaling measurements (MCS) of the ${ }^{6} \mathrm{He}$ decay data were obtained for each detector as a function of PMT bias ( -1800 to $-2400 \mathrm{~V})$ and 584 CFD discriminator settings ( 25 to $200 \mathrm{mV}$ ). These settings were dictated by the need for good statistics and useful signal-to-background ratios (SNR) in the decay data. It must be pointed out that low discriminator settings and high biases were the most unfavorable settings with a SNR $<1$. Conversely, high discriminator settings and low biases tended to eliminate the beta induced scintillator detector signals, effectively detecting only contributions from gamma-background signals.

\section{Data analysis}

As a function of PMT bias, MCS discriminator thresholds, and source distances, 25 decay data sets for each of the two PMTs (detector sides A and B) were analyzed. The analysis of the data collected with this detector (labeled No. 2, side A) for experimental data run 64 is typical of other runs obtained with this detector and is considered here as an example. In this case the PMT bias and discriminator threshold was $-2200 \mathrm{~V}$ and $V_{\mathrm{LO}}=25 \mathrm{mV}$, respectively. Figure 8 displays the $A_{i}$ (counts/dwell time) vs $i$ th dwell-time bin. For analysis, the time assignment was taken at the midpoint of each bin $\left\{t_{i}=0.05+0.1(i-1)\right\}$ in seconds for all $i=1, \ldots, 120$ bins per $12 \mathrm{~s}$ irradiation cycle. The first-time bin $\left(t_{1}=0.05 \mathrm{~s}\right)$ in each cycle coincides with the start of counting after the end of the neutron irradiation time $t_{0}$ (cf. Fig. 7). Visual inspection and a priori physics suggest that the fit function for Fig. 8, $A^{\mathrm{fit}}\left(t_{i}\right)$, should include an exponential beta decay and an additive (but time-rate) constant background. Hence, we choose the following function to fit actual counts in Fig. 8 as

$$
A^{\mathrm{fit}}\left(t_{i}\right)=A_{0} \exp \left[-\left(\ln 2 / \tau_{1 / 2}\right) t_{i}\right]+B
$$

where the parameters, $A_{0}, \tau_{1 / 2}$, and $B$ are the summed initial activity from all irradiation cycles, the ${ }^{6} \mathrm{He}$ decay half-life, and the background count rate (from extraneous sources assumed constant in time). The solid curve (left scale) in Fig. 8 is a fit of $A^{\text {fit }}\left(t_{i}\right)$ to these data, which decline from $\sim 3800$ counts/bin (cpb) at $0.05 \mathrm{~s}$ to $\sim 2500 \mathrm{cpb}$ at late time (background) and from which one infers an initial beta count rate of $\sim 1300 \mathrm{cpb}$. A SNR estimate for these initial counts is $\sim \sqrt{1300} \approx 36$ (alternatively, NSR $\approx 3 \%$ ) and is adequate for a further statistical analysis. Figure 8 also illustrates why 120 cycles of neutron beam pulses were necessary for a reasonable estimate of the initial beta decays: on the right-hand scale one sees $\left\{A^{\mathrm{fit}}\left(t_{i}\right) / 120\right\}$, which peaks at $\sim 32 \mathrm{cpb}$, declines to $21 \mathrm{cpb}$ (background), and roughly yields $11 \mathrm{cpb}$ attributable to initial beta decays, giving a NSR of $\sim 30 \%$ or SNR $\sim 3$. This level of noise limits the precision of the initial beta estimate.

Of these desired fit parameters, the initial activity $A_{0}$ is most important because it relates directly to the neutron sensitivity of the Be detector. The other two parameters are useful in identifying outlying data: for example, if $\tau_{1 / 2}$ differs significantly from the published value of the halflife, one may suspect a problem with the production of a clean ${ }^{6} \mathrm{He}$ beta decay component and detection or the latetime background activity $B$ may not be constant in time. It is not clear from these data where the constant background specifically originates. This component is most likely from long-lived neutron-gamma activation from the hardware and concrete shielding some distance from the Be detector.

A nonlinear, weighted least-squares minimization procedure was chosen to fit the data in Fig. 8 and estimate the three parameters given in Eq. (1). The default GaussNewton (GN) technique from the MINITAB (C) suite of codes was used, but the Levenson-Marquart (LM) also gave virtually identical results. [23,24]. In run 64 , side $A$, the fit values of $A_{0}$ and $B$ were $1367 \pm 75$ and $2502 \pm$ 13 counts/0.1-s bin. The uncertainties quoted for $A_{0}, B$ are at the \pm 2 sigma level (95\% confidence intervals) and are obtained from the regression algorithm. Figure 9 examines the consistency of these measurements over the series of runs for both $\mathrm{A}$ and $\mathrm{B}$ outputs of the $\mathrm{Be}$ detector under test under various settings of the test parameters. Independently plotted in normal probability format are the fitted, decay time estimates $\tau_{1 / 2}$ [Eq. (1)] from this series, altogether 28 runs of which three for side $\mathrm{A}$ (as noted) were regarded as outliers: there were beam on/off issues and distorted decay traces for these data and were thus rejected in the analysis. Figure 9 shows $\tau_{1 / 2}$ (A, B outputs) from the remaining runs which yield remarkably similar, near-Gaussian distributions for $\tau_{1 / 2}$ to each 


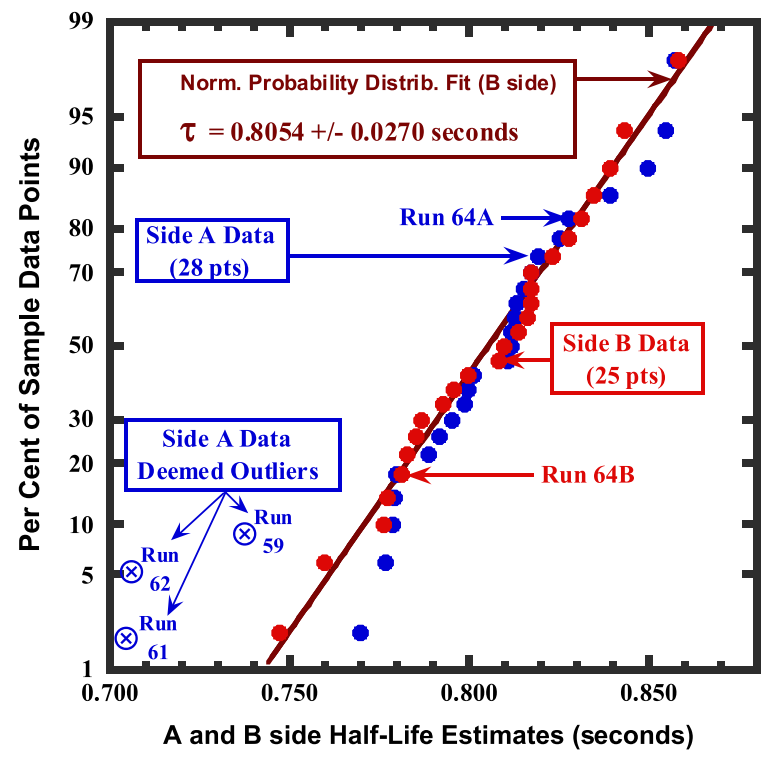

FIG. 9. Two normal probability plots of decay time estimates, for sides $\mathrm{A}$ and $\mathrm{B}$, over 25 experimental runs. The mean and uncertainty is consistent with the accepted decay time of $0.807 \mathrm{~s}$.

other, despite varying test parameters. Combined $\tau_{1 / 2}$ data from both sides A and B yield a half-life mean of $\tau_{1 / 2}=$ $0.8054 \mathrm{~s}$ with a sample standard deviation of $0.027 \mathrm{~s}$. Both estimates bracket the published value of $0.807 \mathrm{~s}$ half-life [8]. We conclude from this analysis, therefore, that the fundamental $n \rightarrow \mathrm{Be} \rightarrow{ }^{6} \mathrm{He} \rightarrow \beta$ decay processes in the Be detector are working as expected.

\section{SUMMARY OF F-FACTOR SENSITIVITY MEASUREMENTS}

The ${ }^{6} \mathrm{He}$ activation decay data for each experimental set of parameters were collected and fitted as above to determine the initial activity $A_{0}$ with subsequent calculation of the $F$-factors. The experiments were performed with 120 or 360 neutron pulses as dictated by needed statistics. Due to limited accelerator availability PMT biases, discriminator settings, and distances from source to detector face were selected. This selection gave $F$ factors with a dynamic range of 18. Most measurements on detector No. 2 were done with a PMT bias at $-2000 \mathrm{~V}$ and discriminator settings between 25 to $200 \mathrm{mV}$ in $25 \mathrm{mV}$ steps. A few additional $F$ factors were determined at -1800 and $-2200 \mathrm{~V}$. The $-2200 \mathrm{~V}$ settings were also used to determine $F$-factors by placing the front face of the detector $35.6 \mathrm{~cm}$ from the source to check for $1 / R_{\text {eff }}^{2}$ (see the Appendix) solid-angle corrections. A good measure of this source to detector distance correction is indicated by agreement with $F$-factors measured at two distances and for each detector's side A and side B with settings shown in Fig. 10 these figures. Each $F$-factor is the average plus

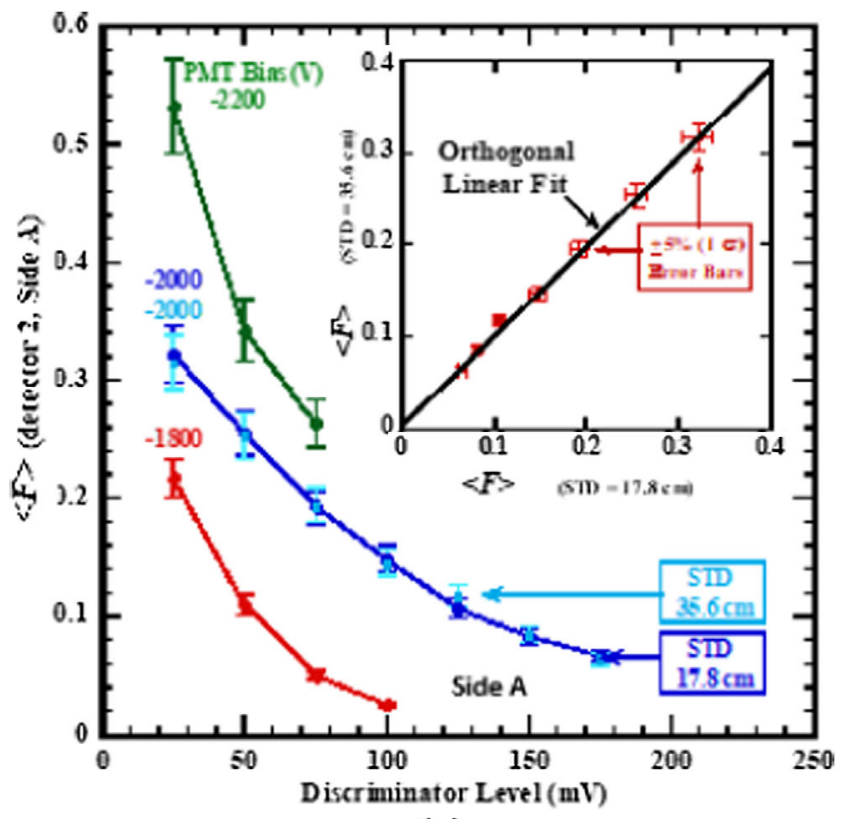

(a)

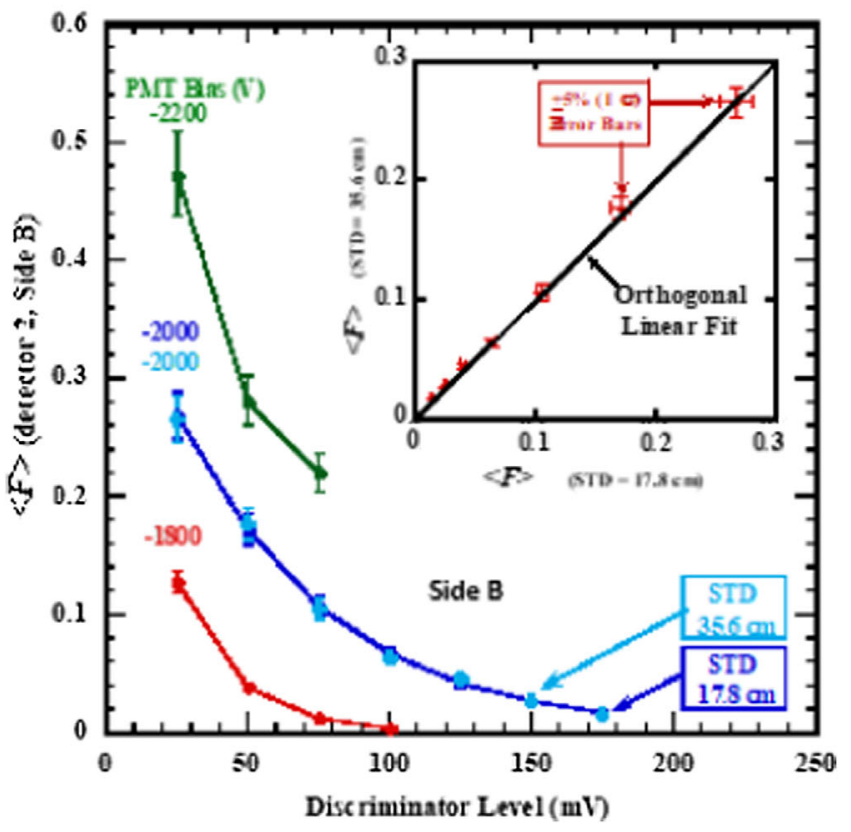

FIG. 10. $F$-factors for Be detector No. 2 side A. The inset shows the correlation plot for $F$ values determined at 17.8 and $35.6 \mathrm{~cm}$ distances. The solid curves are drawn to aid the eye. STD is the source to detector distance.

standard error of the mean. The uncertainties ascribed to each $F$-factor fall in the following categories: (a) counting statistics of the AP measurements, $\pm 3 \%$, (b) background subtraction, $\pm 1 \%$, (c) time-varying beam currents, $\pm 0.1 \%$, (d) deuteron beam energy uncertainties, $\pm 0.1 \%$, (e) solid angle corrections for AP detectors, $\pm 3 \%$, (f) solid angle corrections of Be detector, $\pm 2 \%$, (g) corrections due to fits of the decay data, $\pm 3 \%$, and (h) neutron flux inference from the APT calculation, $\pm 5 \%$. 
This last uncertainty estimate is obtained by the following argument. The front center face of the detector, at 95 degrees and at 17.8 and $35.6 \mathrm{~cm}$ distances respectively, subtended \pm 12 and \pm 8 degrees at the right-front and leftfront detector edges. The DD neutron energy due to these edges for the nearest distance was from 2510 to $2394 \mathrm{keV}$. The ${ }^{9} \mathrm{Be}(n, \alpha)^{6} \mathrm{He}$ cross sections at these extremes were $\leq 5 \%$ above and below the nominal value of $82.5 \mathrm{mb}$ at $2450 \mathrm{keV}$ neutron energy and drove the uncertainty quoted above. Further justification can be observed from the $F$-factor agreement at $35.6 \mathrm{~cm}$ distance compared to $17.8 \mathrm{~cm}$ which subtend different energy spreads. For our Be detector with the front face at 17.8 and $35.6 \mathrm{~cm}$ distance to an isotropic DD source, 218 and $60.5 \mathrm{msr}$ respectively was determined with MCNP modeling. In MCNP the effective solid angle is calculated by taking the product of the beryllium surface area and an average F4 tally; calculated using an isotropic source distribution and a voided geometry. Simple analytical calculations were done to determine the solid angles obtained for each $\mathrm{Be}$ and scintillator layer as a function of source to layer distance. These results were summed, averaged and compare favorably with the MCNP results.

In quadrature the uncertainty in $F$-factors on average is $\pm 7.7 \%$ as indicated in the figures. These uncertainties are not unrealistic considering the good agreement between the $F$-factors determined at $-2000 \mathrm{~V}$ from $25-200 \mathrm{mV}$ discriminator settings and repeated at two distances for both detector No. 2 sides A and B.

\section{FUTURE STUDIES AND CONCLUDING REMARKS}

The performance of the Be detector reported here is ideal in dealing with the time-dependent, mixed radiation field produced in $Z$ MagLIF experiments. To date Be measurements have not been independent but are due to cross calibrations with indium results. The transferring of the $F$-factor (neutron sensitivity) to measure MagLIF DD yields needs further work to determine independent yield measurements. We have identified DD neutron scattering from high $Z$ components near the source that change the neutron spectrum incident on the Be detector implying sensitivity corrections be made. In addition, depending on the location of a detector, neutron activation of nearby support structures or shielding yield prompt gamma-ray signals superimposing the initial ${ }^{6} \mathrm{He}$ decay and compromising the early part of the $0.807 \mathrm{~s}$ half-life decay. We are addressing corrections to proper yield measurements by understanding scattering effects with MCNP modeling. Proper shielding of the Be detector is being explored to remove prompt gamma-ray interference. Since these corrections are very detailed, discussion on the application of our methodology to determine MagLIF DD neutron yields and comparisons of neutron yields from indium activation will be presented in a future publication.
We have made improvements to the associated-particle technique to include the energy spectrum measurement of the ${ }^{3} \mathrm{He}$ contribution from the $D(d, n)^{3} \mathrm{He}$ reaction. This was done by reducing the ${ }^{3} \mathrm{He}$ energy loss by reducing the aluminum foil thickness in front of the SBDs from 2.6 to $0.8 \mu \mathrm{m}$. With the reduction in stopping power from this thinner aluminum filter we now detect the full peak of the ${ }^{3} \mathrm{He}$ particle. This quantity is a direct measurement in a 1:1 ratio of the neutrons produced per ${ }^{3} \mathrm{He}$ particle measured and offers another approach to the determination of neutron yield as opposed to that due to only the proton measurement. The ${ }^{3} \mathrm{He}$ determination will also allow us to measure coincidence neutrons into a neutrontime-of-flight detector for the determination of both DD neutron sensitivity (coulombs/incident neutron) and a neutron impulse response function. This work is currently in progress.

We are exploring using a gamma-ray source, such as Co-60, to precheck the Be detectors for any changes in detection sensitivity. This source will be fixtured at a defined and repeatable location on the surface of the active area of the detectors. The same counting electronics associated with a detector will be used for counting signals from the detector with a chosen bias and discriminator setting, preferably at the highest neutron sensitivity ( $F$-factor). Steady-state counts/unit time will be recorded in an MCS and checked for repeatability every few months. Changes in the counting due to aging of the scintillators, gain changes in the PMTs or discriminator levels in the CFDs will necessarily require calibrations at IBL.

A preliminary study with DT neutrons suggests that the Be detector as designed can be used to measure DT neutron yields, an important future consideration at $Z$. These studies will also be presented in a future publication.

\section{ACKNOWLEDGMENTS}

Thanks to Ken Moy (Santa Barbara, California), Irene Garza, Robert Buckles, and Brent Davis (Las Vegas, Nevada) at Nevada National Security Sites (NNSS) for manufacturing the detector components and characterizing the fidelity of two Be detector PMTs. Sandia National Laboratories is a multi-mission laboratory managed and operated by National Technology and Engineering Solutions of Sandia, LLC., a wholly owned subsidiary of Honeywell International, Inc., for the U.S. Department of Energy's National Nuclear Security Administration under Contract No. DE-NA-0003525. The views expressed in the article do not necessarily represent the views of the U.S. Department of Energy or the United States Government.

\section{APPENDIX}

A general expression [25-27] for the activation of an elemental sample of mass $\mathrm{M}$ and atomic weight $A_{w}$ from a 
steady state neutron flux $\varphi\left(\mathrm{n} / \mathrm{cm}^{2}\right.$-sec $)$ with a kinetic energy $E(\mathrm{MeV})$ is given by Eq. (A1) as

$$
\begin{aligned}
(C-B)= & \phi \varepsilon_{a} \varepsilon_{b} \varepsilon_{c} \varepsilon_{d} M N_{A} \sigma(E) \\
& \times\left[\left(1-e^{-\lambda t_{0}}\right)\left(e^{-\lambda t_{1}}-e^{-\lambda t_{2}}\right)\right] /\left(\lambda A_{W}\right),
\end{aligned}
$$

where the quantities are as follows: $\varepsilon_{a}$ is the natural abundance, in our case, of ${ }^{9} \mathrm{Be} ; \varepsilon_{b}$ is the neutron detection efficiency of the detector; $\varepsilon_{c}$ is the self-attenuation of neutrons from the detector casing; $\varepsilon_{d}$ is the branching ratio of ${ }^{6} \mathrm{He}$ beta decay; $M$ is the amount of Be mass (gms); $N_{A}$ is Avogadro's number; $\sigma(E)$ is the energy dependent neutron reaction cross section for ${ }^{9} \mathrm{Be}\left(n,{ }^{4} \mathrm{He}\right){ }^{6} \mathrm{He} ; A_{w}$ is the atomic weight of activating material; $\lambda$ is the decay constant $\ln 2 / \tau_{1 / 2}$ of the sample isotope where $\tau_{1 / 2}$ is the half-life; $t_{0}$ is the neutron irradiation time (not an instantaneous time pulse); $t_{1}$ is the starting time for counting the decay activity (counts/unit time) after the end of $t_{0}$; and $t_{2}$ is the ending time for counting the decay activity after $t_{0}$. The ${ }^{6} \mathrm{He}$ beta decay activation total counts between time interval of $t_{1}$ and $t_{2}$ is given by $C$ (counts) corrected for background $B$. Background counts $B$ occur over the same time interval $t_{1}$ to $t_{2}$. Equation (A1) can be rearranged into the following two expressions-(A2) and (A3)—as follows:

$$
F=(C-B) \lambda /\left[\phi\left(1-e^{-\lambda t_{0}}\right)\left(e^{-\lambda t_{1}}-e^{-\lambda t_{2}}\right)\right],
$$

which also equals

$$
F=\varepsilon_{a} \varepsilon_{b} \varepsilon_{c} \varepsilon_{d} M N_{A} \sigma(\mathrm{E}) / A_{W} .
$$

Note that both expressions are composed of quantities that are previously known or measurable from experiment. The quantity of choice here is defined by expression (A2) since these are quantities easily measured. Expression (A2) defines a detector's neutron detection sensitivity (counts/ per incident neutron $/ \mathrm{cm}^{2}$ )-sec. We call this expression the $F$-factor. The most important part of the expression is the inference on the production of neutron flux $\phi\left(\mathrm{n} / \mathrm{cm}^{2}-\mathrm{sec}\right)$ incident on the detector. This is defined as

$$
\phi=N /\left(A_{\text {eff }} t_{0}\right),
$$

where $A_{\text {eff }}\left(\mathrm{cm}^{2}\right)$ and $R_{\text {eff }}(\mathrm{cm})$ are the effective area subtended and effective distance from a volumetric detector to a source. Both quantities are obtained from MCNP modeling to determine the effective subtending solid angle $\Omega_{\text {eff }}$. The effective area $A_{\text {eff }}$ is given by

$$
A_{\text {eff }}=\Omega_{\text {eff }}\left(R_{\text {eff }}\right)^{2} .
$$

The total neutrons $N$ incident on the detector is determined by the associated-particle technique [25-27]. This technique here is applied to the DD fusion reaction to determine from known parameters a produced or inferred neutron fluence. The technique employs calculational and experimental procedures and makes important use of previously studied $D(d, n)^{3} \mathrm{He}$ and $D(d, p) T$ reactions as functions of deuteron kinetic energy. The calculational part is approached as follows. Imagine the production of neutrons from the $D(d, n)^{3} \mathrm{He}$ reaction at a certain deuteron energy and emitted at a lab angle relative to the impinging deuteron on a stationary deuterated target. The calculation of the number of neutrons, protons, tritium ions, or ${ }^{3} \mathrm{He}$ produced, into a laboratory detector solid angle depends on the kinetic energy of the deuterons, differential cross section, loading ratio of deuterium in the target, and the stopping power of the deuterated target compound. The general expression describing this process is

$$
d Y / d \Omega=n \int_{E_{m}}^{0}\{[d \sigma(E) / d \Omega] /(d E / d x)\} d E
$$

in units of number of $\mathrm{n},{ }^{3} \mathrm{He}, p$ or $T$ produced per solid angle (msr) of the detecting instrument per total deuteron particles incident on the target converted to charge $(\mu \mathrm{C})$. The target used here is a deuteron loaded erbium with a thickness $x\left(\mu \mathrm{gm} / \mathrm{cm}^{2}\right)$, density $\left(3.94 \mathrm{gms} / \mathrm{cm}^{3}\right)$, and the number of deuteron atoms per erbium atoms $n$ (loading ratio) was typically 1.96 to 1.98 . The target thickness was chosen to completely stop a deuteron with a kinetic energy up to $250 \mathrm{keV}$. The differential cross section $d \sigma(\mathrm{E}) / d \Omega$ is in the laboratory frame for either the $(d, n),\left(d,{ }^{3} \mathrm{He}\right),(d, p)$, or the $(d, T)$ reaction, the stopping power $d E / d x$ in units of $\mathrm{keV} /$ atoms $/ \mathrm{cm}^{2}$ of the $\mathrm{Er}+n d$ target compound as a function of deuteron energy, and the maximum deuteron energy $E_{m}$ [28]. The stopping power is prescribed by a parametrized expression as a function of the deuteron kinetic energy for target-deuteron compound [29]. The calculations leading to the integral expression are done here from first principles: transformations of lab to $\mathrm{cm}$ back to lab of the kinematics and $\mathrm{cm}$ to lab differential DD cross section [30], stopping powers calculated for deuterons on $\operatorname{Er} D_{n}$ targets, and numerical integration of Eq. (A6). Calculations for Eq. (A6) were done only for the proton and neutron portion of the reactions. In these experiments, the measurement of the proton yield and neutron yields are summarized in the main text. Calculations for protons were done at 110 and 165 degrees. These numbers are 43.43 and $58.85 \mathrm{p} / \mathrm{msr} / \mu \mathrm{C}$ respectively. Since the objective of the Be calibrations is to conduct $F$-factor measurements with DD inertial confinement fusion (ICF) neutron energies of $2.45 \mathrm{MeV}$ a nominal angle was chosen for the placement of the Be detector centered relative to the neutron direction. The kinematic portion of the calculations indicates that to be a nominal 95 degrees. A legitimate question arises as to what are the average spread of neutron energies across a detector and leading to additional uncertainties in the $F$-factor determination. This question is addressed in the 
main text for the specific geometrical construction of a detector. At the 95 degree angle the calculated neutron yield is $42.07 \mathrm{n} / \mathrm{msr} / \mu \mathrm{C}$. The quantities $\mathrm{n} / \mathrm{msr} / \mu \mathrm{C}$ and $\mathrm{p} / \mathrm{msr} / \mu \mathrm{C}$ are each multiplied by their respective detector subtended solid angles but the ratio of these cancel out the units $/ \mu \mathrm{C}$. The cancellation of charge occurs because the same charge is on the target contributing, in a $1: 1$ ratio, to the neutron and proton $(d, n)$ and $(d, p)$ reactions respectively. As a calculational check the neutron yield at 0 degrees is $104.0 \mathrm{n} / \mathrm{msr} / \mu \mathrm{C}$ confirming the expected yield being about twice that of 95 degrees [31]. Also, the neutron fluence, inferred from two independent proton measurements at two angles, should be equal with small differences due to calculational and experimental uncertainties confirming this calculational approach.

[1] S. A. Slutz, M. C. Hermann, R. A. Vesey, A. B. Sefkow, D. B. Sinars, D. C. Rovang, K. J. Peterson, and M. E. Cuneo, Pulsed-power-driven cylindrical liner implosions of laser preheated fuel magnetized with an axial field, Phys. Plasmas 17, 056303 (2010).

[2] C. L. Ruiz, R. J. Leeper, A. Schmidlapp, and G. W. Cooper, Absolute calibration of a total yield indium activation detector for DD and DT neutrons, Rev. Sci. Instrum. 63, 4889 (1992).

[3] K. D. Hahn et al., Fusion-neutron measurements for magnetized liner inertial fusion experiments on the $Z$ accelerator, J. Phys. Conf. Ser. 717, 012020 (2016).

[4] M.S. Rowland and J.C. Roberson, A novel neutron detector for measuring the output of pulsed sources, Radiat. Eff. 96, 21 (1986).

[5] T. J. Murphy, A practical beryllium activation detector for measuring DD neutron yield from ICF targets (LA-UR96-1649). United States, in the 11th High Temperature Plasma Diagnostic Conference, Monterey, California, 1996.

[6] A. Talebither, S. V. Springham, R. S. Rawat, and P. Lee, Beryllium neutron activation detector for pulsed DD fusion sources, Nucl. Instrum. Methods Phys. Res., Sect. A 659, 361 (2011).

[7] D. A. Brown, M. B. Chadwick, R. Capote, and A.C. Kahler, Nucl. Data Sheets 148, 1 (2018).

[8] D. R. Tilley, C. M. Cheves, J. L. Godwin, G. M. Hale, H. M. Hofmann, J. H. Kelley, C. G. Sheu, and H. R. Weller, Energy levels of light nuclei $A=5,6,7$, Nucl. Phys. A 708, 3 (2002).

[9] J. D. Styron, G. W. Cooper, C. L. Ruiz, K. D. Hahn, G. A. Chandler, A. J. Nelson, J. A. Torres, B. R. McWatters, K. Carpenter, and M. A. Bonura, Predicting the sensitivity of the beryllium/scintillator layer neutron detector using Monte Carlo and experimental response functions, Rev. Sci. Instrum. 85, 11E617 (2014).

[10] R. D. Evans, The Atomic Nucleus (McGraw-Hill, New York, 1955), pp. 536 and 627.
[11] W. M Stacey, Nuclear Reactor Physics 2nd ed. (Wiley-VCH Verlag GmbH and Co., KGaA, Weinham, 2007), p. 316.

[12] A. A. Harms and D. R. Wynean, Mathematics and Physics of Neutron Radiography (D. Reidel Publishing Co., Boston, 1986), p. 14ff.

[13] A. B. Chilton, J. K. Shultis, and R. E. Faw, Principles of Radiation Shielding (Prentice-Hall, Englewood Cliffs, NJ, 1984), p. 145.

[14] F. H. Attix, Introduction to Radiological Physics and Radiation Dosimetry (Wiley, New York, 1986).

[15] MCNP_A general Monte Carlo N-particle transport code, Version 5, Report No. LA-UR-03-1987, Los Alamos National Laboratory, 2008.

[16] J. B. Vise and B. M. Rustad, Electron-neutrino angular correlation in the decay of $\mathrm{He}^{6}$, Phys. Rev. 132 (1963).

[17] http://www.crystal.saint-gobain.com for summary of scintillator BC-404 specifications.

[18] http://www.hamamatsu.com/us/em/3001.html for information on Hamamatsu PMT products.

[19] R. B. Theus, W. I. McGarry, and L. A. Beach, Angular distributions and cross-section ratios for the reactions ${ }^{2} H(d, n){ }^{3} \mathrm{He}$ and ${ }^{2} H(d, p){ }^{3} H$ below $500 \mathrm{keV}$, Nucl. Phys. 80, 273 (1966).

[20] http://www.ortec-online.com for descriptions of ORTEC NIM and data acquisition modules used in our application exclusively.

[21] http://www.ezag.com for information and contact information on the Am-241 alpha source.

[22] http://www.bellmw.com/manufacturer/thinksrs.com for power supply and gate generator details.

[23] http://www.minitab.com/en-us/ for availability of MINITAB software.

[24] CRC Standard Probability and Statistics Tables and Formulae, edited by W. H. Beyer (CRC Press, Boca Raton, FL, 1991).

[25] R. J. Leeper, K. H. Kim, D. E. Hebron, N. D. Wing, and E. Norbeck, Intense lithium ion beam diagnostic using the inverse nuclear reaction $D\left({ }^{7} \mathrm{Li}, n\right)^{8} \mathrm{Be}$, Nucl. Instrum. Methods Phys. Res., Sect. B 24/25, 695 (1987).

[26] G. W. Cooper and C. L. Ruiz, NIF total neutron yield diagnostic, Rev. Sci. Instrum. 72, 814 (2001).

[27] L. Ruby and R. B. Crawford, Anisotropy factors for the determination of total neutron yield from the $D(d, n) \mathrm{He}^{3}$ and $T(d, n) \mathrm{He}^{4}$ reactions, Nucl. Instrum. Methods $\mathbf{2 4 ,} 413$ (1963).

[28] J. F. Ziegler, The Stopping and Ranges of Hydrogen Ions in Matter (Pergamon Press, New York, 1977).

[29] D. Malbrough, D. K. Brice, D. F. Cowgill, J. A. Borders, L. A. Shope, and J. M. Harris, Deuteron stopping cross sections in transition metal hydrides, Nucl. Instrum. Methods Phys. Res., Sect. B 28, 459 (1987).

[30] A. Michalowicz, Kinematics of Nuclear Reactions, edited by R. A. Giles (Iliffe Books Ltd., London, 1967).

[31] H. Lisken and A. Paulsen, Neutron production cross sections and energies for the reactions $T(p, n)^{3} \mathrm{He}$, $D(d, n)^{3} \mathrm{He}$, and $T(d, n)^{4} \mathrm{He}, \mathrm{Nucl}$. Data Tables 11, 569 (1973). 